\title{
Infant and young child feeding practices on Unguja Island in Zanzibar, Tanzania: a ProPAN based analysis
}

JOYCE KINABO ${ }^{1}$, AKWILINA W. MWANRI ${ }^{*}$, PETER MAMIRO ${ }^{1}$, NYAMIZI BUNDALA ${ }^{1}$, JANET PICADO², KISSA KULWA ${ }^{1}$, JOHN MSUYA ${ }^{1}$, JULIUS NTWENYA ${ }^{3}$, ANETH NOMBO ${ }^{4}$, REHEMA MZIMBIRI ${ }^{4}$, FATMA ALLY ${ }^{5}$, ASHA SALMINI ${ }^{5}$, ELIZABETH MACHA ${ }^{6}$ and EDITH CHEUNG $^{7}$

${ }^{1}$ Department of Food Technology, Nutrition and Consumer Sciences, Sokoine University of Agriculture, P.O. Box 3006, Chuo Kikuu, Morogoro, Tanzania

${ }^{2}$ Altos de Santo Domingo 31 C, Managua, Nicaragua

3 University of Dodoma, Dodoma, Tanzania

${ }^{4}$ Tanzania Food and Nutrition Centre, Dar-es-Salaam, Tanzania

${ }^{5}$ Ministry of Health and Social Welfare, Zanzibar; Tanzania

'United Nations Children's Fund, Dar es Salaam, Tanzania

7United Nations Children's Fund, New York, United States of America

\begin{abstract}
Background: Undernutrition in children has remained a challenge despite the success achieved in reduction of other childhood diseases in Zanzibar. Most empirical studies on infants and young child feeding (IYCF) have examined nutritional value of foods fed to the children in terms of energy and micronutrient content. Little is known on the role of culture, traditions and social norms in influencing IYCF practices. This study aimed at assessing the existing IYCF practices and socio-cultural factors influencing these practises using ProPAN tool to get an in-depth understanding of the mothers' practices with respect to exclusive breastfeeding and infant and young child feeding.

Methods: A cross sectional study involving mothers/caregivers was conducted using semi-structured interviews and opportunistic observations on Unguja Island, Zanzibar. A random selection of 36 Shehias and 213 households with children 0-23 months old was done proportional-to-size of Shehias and children. Quantitative and qualitative data were collected using the ProPAN tool and embedded software for analyses.

Results: A total of 213 of mothers/caregivers were involved in the study. Almost all new-borns were fed colostrum (96\%). Proportion of mothers who initiated breastfeeding within one hour after giving birth was $63 \%$. About $24 \%$ of the women practiced exclusive breastfeeding and $21 \%$ of the children were given pre-lacteal feeds 2-3 days after birth. The mean age of introduction of complementary foods was four months. Responsive feeding was not practiced by some mothers as $31 \%$ of the children $12-23$ months of age were left to eat alone without any support from caregivers. The common reasons for non-adherence to the recommended feeding practices were related to income and socio-cultural norms which are strong in the community and supported by other family members.

Conclusion: Infant and young child feeding in Unguja was suboptimal. Inadequate care practices, use of pre-lacteals, early introduction of complementary foods, low rate of exclusive breastfeeding and low meal frequency were among the common practices. The main causes of the observed feeding practice were related to socio-cultural norms and beliefs. There is a need to strengthen IYCF education into the antenatal clinic regime.
\end{abstract}

Keywords: infants, children, feeding practices, ProPAN, Zanzibar

\section{Introduction}

Malnutrition in Zanzibar has remained a great challenge despite the success achieved in reduction of other childhood diseases such as measles and malaria. On average stunting levels in children below five years of age is 30\%, acute malnutrition is $12 \%$, and anaemia is $68 \%$. These levels are unacceptably high (TDHS, 2011). However, there are regional variations whereby prevalence of stunting in North Unguja and Pemba North is $40 \%$ and South Unguja and Urban West the rates are $29.1 \%$ and $19.6 \%$, respectively (TDHS, 2011). Poor feeding practices during infancy and childhood could be a major contributing factor to the observed scenario. Inadequate food intake among

*Correspondence E-mail: akwmwanri@hotmail.com 
children results in undernutrition, impaired cognitive and social development, poor school performance and reduced productivity in later life (Caulfield et al., 2006).

Optimal breastfeeding behaviours and practices such as exclusive breastfeeding for 6 months and continued breastfeeding to 24 months with appropriate complementation can help to reduce childhood morbidity and mortality (WHO 2009; Montee \& Jeewon, 2014). It also has a significant benefit for both infants and mothers and these include reduced morbidity, improved growth and nutritional status, improved cognitive and motor development and generally has a contraceptive effect for the mother in the early months after delivery (Dewey et al., 2001; Stuebe, 2009; Motee \& Jeewon, 2014).

Most empirical studies on IYCF have examined foods that are being fed to the children in terms of energy and nutrient content and safety (Mamiro et al. 2005; Kulwa et al., 2015). However, the influences of culture, traditions and social norms on IYCF have not been adequately examined. In addition, there is limited understanding of the contribution of other household members such as spouses or mother in laws on ensuring adequate IYCF. Adoption of innovations to improve IYCF and nutrition status of children requires understanding of factors influencing behavioural and caring practices. This necessitated use of comprehensive tools in identifying the existing IYCF practices in Zanzibar and assessing the influence of institutional, community, social, cultural, familial and individual factors.

\section{Materials and Methods}

\section{Study area}

This study was carried out in October 2012 to March 2013 in Unguja Island, Zanzibar, which is part of the United Republic of Tanzania. Unguja is a hilly island located in the Southern half of the Zanzibar Archipelago, in the Indian Ocean and is surrounded by about 50 smaller islands and islets. It has an overall area of about $1,666 \mathrm{~km}^{2}$. Unguja is divided into three regions namely north, south and urban west. Each region is divided into two districts making a total of six districts. The districts are further subdivided into varying numbers of Shehias, making a total of 198.

\section{Study design and population}

The study employed repeated cross-sectional design. The first phase involved situation analysis, and the second phase involved testing the recommended practices. The study population consisted of children 0-23 months old and their mothers or caregivers. Other participants who facilitated the process of data collection included family members (in-laws, men/spouses, and siblings), health personnel, and community leaders (Shehas). In Zanzibar close to half of the population ( $48 \%$ ) live below the basic needs poverty line (shelter, clothing and food) and 13\% below the food poverty line. In addition, high dependence ratio (0.95) and low level of education (literacy rate $65 \%$ ) partly contribute to poor socio-economic status of the population. Female headed households accounted for $30 \%$ of the total households and average household size is 5.3 (Mamiro et al. 2005).

\section{Sample size and sampling procedure}

A list of all Shehias $(n=198)$ from the six districts was prepared and 36 Shehias were selected randomly proportional-to-size of their number in the district. Also the number of households sampled was determined proportion to number of households in each Shehia. In each selected Shehia, participants were purposively identified based on the list of households with mothers of children below two years of age that was prepared by the Sheha. After all the participants were identified random selection was carried to obtain the required number of households in each respective Shehia. Through this process a total of 213 households were selected.

Sample size was calculated from the following Fisher's formula, $N=Z^{2}\left(p^{*} q\right) / d^{2}$. Where: $n=$ desired minimum sample size; $Z$ = the standard normal deviate corresponding to $95 \%$ Confidence 
Interval; $\mathrm{p}=$ the proportion of an indicator measured (In this case prevalence of stunting); $\mathrm{q}=1-\mathrm{p}$; $\mathrm{d}=$ degree of accuracy or desired precision. Taking the prevalence of stunting in Zanzibar as $26.7 \%$, $Z$ statistic corresponding to $95 \%$ confidence interval for a two-tailed test as 1.96 , and degree of accuracy at 0.05, the sample size was 300 .

\section{Data collection}

In-depth interviews using a semi structured questionnaire contained in a ProPAN field manual were conducted with mothers/caregivers to gather information on IYCF and child care practices (PAHO, 2004). The information collected included but not limited to socio-demographic characteristics, socio-economic status, breast feeding and complementary feeding practices, child care practices, hygiene/sanitation, health/nutrition knowledge and HIV related aspects. The interviews were conducted at the respondent's home to allow the interviewer to make opportunistic observation on feeding practices such as interaction of the caregiver and the child during meal time, assess the consistency, food preparation, general cooking practices and hygiene.

To understand mother's perceptions, beliefs and opinions regarding child-feeding practices, a sub sample of 13 mothers/caregivers with children of age between 6-11 months $(n=7)$ and 12-23 months $(\mathrm{n}=6)$ was interviewed using a semi-structured questionnaire with open ended questions. In addition, 15 other mothers with children aged 6-23 months (7 with children of age 611 and 8 with children of age 12-23 months according to the ProPAN guidelines) were selected randomly among those who were interviewed (213 mothers) and each was observed during a single feeding episode. Observation elements included food consistency, food preparation, caregiver/child interaction during feeding, cooking practices, and general hygiene in the home. The inclusion criteria were caretaker willing to participate and give verbal or written consent and healthy child. Exclusion criteria were caretaker not willing to participate and having a sick child. In the event caretakers had twins, the younger twin was taken measurements but in the feeding activity all twins were involved.

\section{Data analysis}

Data were entered into a database and analysed using the ProPAN software (PAHO 2004). Descriptive statistics, such as mean, standard deviation, median and frequencies and confidence intervals were determined. Qualitative data from opportunistic observations and semi-structured interviews were compiled manually in matrices using ideal feeding practices (PAHO 2004). The identified IYCF practices and context in which they are practiced were compared with the World Health Organization recommendations to determine their adequacy (WHO, 2009).

\section{Ethical considerations}

Ethical clearance was granted by Zanzibar Medical Research Ethical Committee. The research protocol was explained to administrative officials of the districts and selected Shehias. Parents of eligible children were informed of the objectives and activities of the research. Parents who agreed to participate provided verbal informed consent. Caregivers of children who were identified by the researchers as malnourished and those who were reported to be ill during the survey were advised to report to health facilities for standard treatment and or rehabilitation.

\section{Results}

\section{Socio-demographic characteristics}

A total of 213 mothers were interviewed at the households with a response rate of $99 \%$. The mean age (and standard deviation) of the surveyed children was $12.5( \pm 5.4)$ months. Categorization of the studied children by sex showed that about $46 \%$ were males. Similarly, the surveyed households had a total of 1326 household members with an average household size of $6.2 \pm 2.6$ members. Out of these 349 were children of age less than five years; an average of $1.6(S D=0.8)$ children per 
household. Almost all of the caregivers interviewed were mothers of the studied children. The average age of the caregivers was $30(S D=6.5)$ years. Information on marital status showed that $5 \%$ of the caregivers were single and $4.7 \%$ were separated/divorced/widowed. More than half of the caregivers (56\%) had attained secondary education, $15 \%$ had never gone to school and $18 \%$ did not know how to read and write. Only $23 \%$ of mothers had done paid work in the past seven days prior to the survey. Among those who did some economic activities, $79.6 \%$ were food vendors and $13.3 \%$ were employed and doing office work (Table 1).

Table 1: General characteristics of mothers/caregivers

\begin{tabular}{llll}
\hline Variable & Response & Frequency & Percentage \\
\hline Sex & Male & 97 & 45.5 \\
Marital status & Female & 116 & 54.5 \\
& Single & 11 & 5.2 \\
& Married/with partner & 192 & 90.1 \\
Education level (years) & Separated/divorced/widowed & 10 & 4.7 \\
& None & 32 & 15.0 \\
& Incomplete primary education (1-6) & 28 & 13.1 \\
& Complete primary education (7) & 30 & 14.1 \\
& Secondary (8-13) & 119 & 55.9 \\
Occupation & Post-secondary (214) & 4 & 1.9 \\
& Food vendor & 39 & 79.6 \\
& Office work & 7 & 13.3 \\
& Peasantry & 3 & 6.1 \\
\hline
\end{tabular}

\section{Breastfeeding}

Breastfeeding was practiced by almost all mothers. About $97 \%$ of the mothers reported that they had ever breastfed their children prior to the survey and $63 \%$ initiated breastfeeding within an hour after giving birth (Fig. 1). Colostrum was fed to $96 \%$ of the new-born babies; nevertheless $21 \%$ of the babies were given pre-lacteal feeds (honey (89\%) and water (34\%)), two to three days after they were born. Exclusive breastfeeding was reported by only $24 \%$ of the mothers. Only $47 \%$ of the mothers reported to have received breastfeeding support during the first three days after delivery. In addition, $88 \%$ of the children of age less than two years were still being breastfed during the time of the survey, out of which $5.8 \%$ were 20 to 23.9 months old.

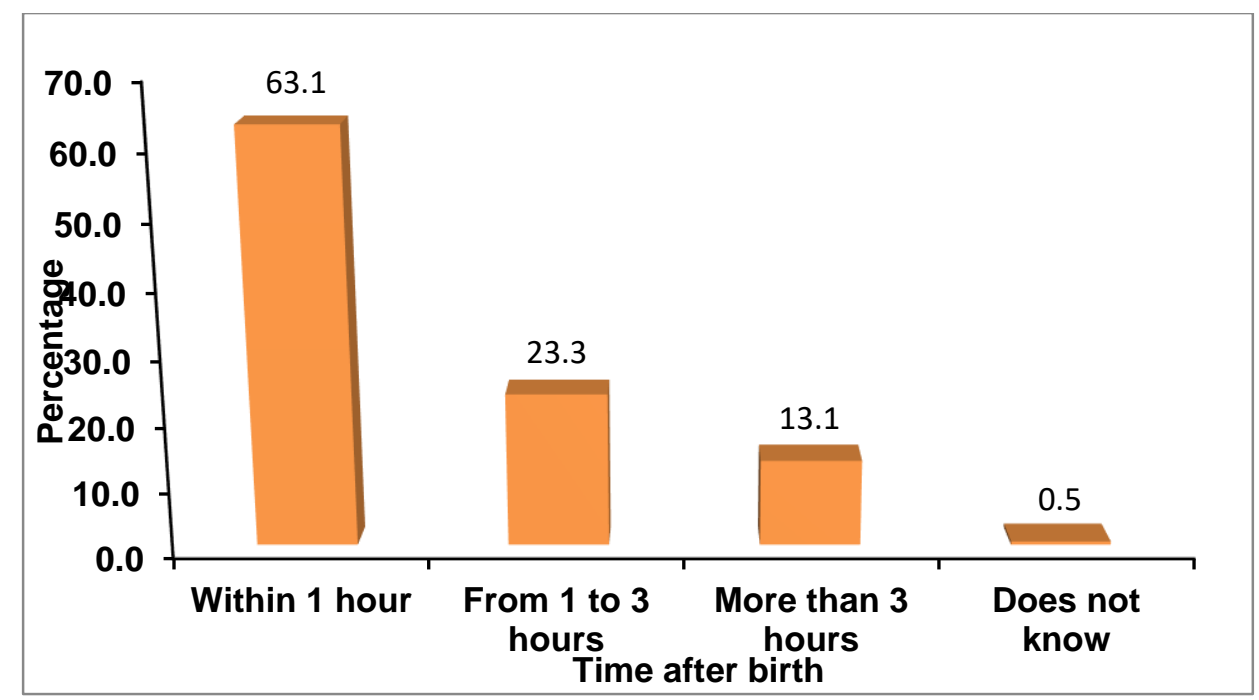

Figure 1: Time at the initiation of breastfeeding 


\section{Complementary feeding}

The mean age at which complementary foods were introduced to children was 3.9 (SD 1.8) months. About $89 \%$ of the children were fed complementary foods 24 hours preceding the survey. The majority of caregivers ( $82 \%$ ) perceived that the appetite of their children was satisfactory and the amount of food consumed was adequate (Table 2). Some of the children (31\%) of 12-23 months of age were served on a separate plate and left to feed themselves all the time during the main meal without any supervision or responsive feeding. For mothers who practised responsive feeding, encouraged their children to eat either by talking and singing to them or by offering another food when the child refused to eat the one that was prepared.

Table 2: Complementary feeding practices

\begin{tabular}{|c|c|c|c|}
\hline Variable & Response & Frequency & Percentage \\
\hline \multirow[t]{3}{*}{ Perception on child's appetite } & Eat well & 89 & 43.6 \\
\hline & Eat too much & 75 & 36.8 \\
\hline & Eat a little & 40 & 19.6 \\
\hline \multirow[t]{5}{*}{ Who decides what the child should eat } & Mother & 184 & 86.9 \\
\hline & Father & 14 & 6.6 \\
\hline & Aunt & 3 & 1.4 \\
\hline & Grandmother & 3 & 1.4 \\
\hline & Does not apply & 9 & 3.8 \\
\hline Response feeding & Child ate all the food & 146 & 82 \\
\hline \multirow[t]{4}{*}{ Child self-fed $(n=98)$} & All the time & 30 & 30.6 \\
\hline & Half of the time & 29 & 29.6 \\
\hline & Little bit of the time & 39 & 39.8 \\
\hline & $\begin{array}{l}\text { Encourage child during main } \\
\text { meal yesterday }\end{array}$ & 86 & 40.2 \\
\hline \multirow[t]{4}{*}{ Type of encouragement } & Encourage verbally & 52 & 59.8 \\
\hline & Offered another food & 22 & $25 \cdot 3$ \\
\hline & Ordered strongly & 7 & 8.0 \\
\hline & $\begin{array}{l}\text { Modelled eating (using } \\
\text { toys) }\end{array}$ & 2 & 2.3 \\
\hline Received information on child feeding & Yes & 56 & 26.3 \\
\hline \multirow[t]{8}{*}{ Source of information on child feeding } & Health facility & 110 & 48.5 \\
\hline & Radio & 50 & 22.0 \\
\hline & Community health worker & 34 & 15.0 \\
\hline & Neighbour/Friend & 12 & $5 \cdot 3$ \\
\hline & Television & 10 & 4.4 \\
\hline & Family member & 8 & $3 \cdot 5$ \\
\hline & Do not remember & 2 & 0.9 \\
\hline & Religious institution & 1 & 0.4 \\
\hline \multirow[t]{4}{*}{ Supplements } & Vitamin A & 192 & 90.1 \\
\hline & Iron & 11 & $5 \cdot 3$ \\
\hline & $\begin{array}{l}\text { Multivitamin and mineral } \\
\text { supplement }\end{array}$ & 5 & 2.3 \\
\hline & Lipid nutrient supplement & 6 & 2.8 \\
\hline
\end{tabular}

About $25 \%$ of the mothers reported to have accessed information on child feeding at least once during one month prior to the survey. The main sources of information were health facilities (48.5\%) and radio (22\%). Decision on what to feed the child was predominantly done by the mother (87\%). In some cases, a father, grandmother or the aunt could also influence on what the child was given to eat. Majority of mothers gave their children vitamin A supplement (90\%) and very few gave iron, multivitamin, mineral and lipid based nutrient-supplements. The assessment of the eating pattern during illness showed that children received less foods and fluids before they got 
sick. Some mothers offered their children less amount of breast milk (35\%) or non-breast milk liquids (24\%) because of child's refusal to feed. After the illness, most of the children (72\%) reverted to consuming the same amount of food as when they were not sick.

\section{Attitude and practice of ideal feeding practices}

A total of 13 mothers/caregivers were interviewed to assess their knowledge, attitude and practice of ideal feeding practices. There were divided opinions among mothers on the issue of initiation of breastfeeding within the first hour after delivery. Mothers who initiated breast feeding within one hour after delivery indicated that it is important to breastfeed the baby immediately after delivery before it starts feeling hungry. For those who initiated breastfeeding later than one hour after delivery provided various reasons for their practice. These included failure of milk to start flowing immediately after giving birth; mothers taking time to clean themselves and the baby in preparation for breast feeding; the baby was too weak to start suckling immediately so they had to wait for some time for the baby to get stronger; some mothers believed that before the baby was breastfed; it must be given honey to taste the sweetness of the world. Therefore, some mothers usually wait for honey to be obtained and given to the baby before initiating breast feeding.

Mothers who reported to have given their infants pre-lacteal feeds (honey, herbal extracts, glucose solution, sugar, salt solution and infant formula) were advised by other women while in the maternity room or her mother, aunt, grandmother. Some of the mothers did not know the reasons for such a practice. In fact, one mother admitted that she had given honey to her baby after delivery out of ignorance and vowed never to do the same again if she gets another baby. Some mothers gave their children infant formula to satisfy a frequent crying baby. This is because they believed that mother's milk was not enough for the baby. Regarding feeding colostrum to the babies, almost all women gave their children colostrum because they believed that it is good for the baby. Mothers felt that they were obliged to practice what they had been told about the advantages of colostrum by health workers at the health facilities (protective effects and good nutrition for a child). Some mothers admitted that culturally, a child must be given the mother's milk and nothing else because it also has medicinal effects. However, few who did not give colostrum believed that it may cause stomach discomfort to the child.

The ideal practice recommends that all infants and young children are breastfed on demand during the day and night. This recommendation was implementable by most mothers because they were house wives and therefore not working outside the home. Reasons for this practice included, prevention of the mother from becoming pregnant too soon after delivery and helps to avoid frequent crying of the baby. For mothers who did not breastfeed on demand believed that by following a regular schedule of feeding it allows the mother to produce enough milk to satisfy the baby. Working mothers could not implement this practice because they were occupied by their businesses and the environment was not conducive for breast feeding. Few mothers practiced exclusive breastfeeding because they believed that mother's milk is the best food for a baby and sufficient to meet their requirements. Some of the reasons given for early initiation of complementary feeding and giving water to the child were: (i) the child's thirst cannot be quenched by breast milk alone so the child should be given water. They believed that giving water is necessary to avoid hiccups and thirst. One woman said "I cannot stop giving water unless I get a medicine to stop hiccup and abdominal discomfort" (ii) Feels that it is not possible to avoid giving a baby what you eat because "if a child is looking at you while you are eating it is a sign that the child is begging; only that it cannot speak". "This has been a practice for a long time even for us (mother) that we were given other foods. For them they could not understand why they should practice differently or not do the same to her baby!?" Some of the perceived reasons by mothers for early complementation were frequent child crying as was not satisfied with the breast milk; child refusing breast milk; mother not eating adequately to produce sufficient milk due to lack of financial support from spouse/partner. 
Those who started complementary feeding at 6 months said they were advised by health personnel that at that age, the child needs extra food apart from breast milk. Mothers believed that extended breast feeding for up to 24 months helps to avoid pregnancy and a Religious believes (Moslem) also emphasize the same. Those who stopped to breastfeed before 24 months felt that the child has grown up enough and therefore can eat well. Others felt that prolonged breast feeding could 'spoil' a child and cause him/her to refuse eating. Much of the foods used for feeding infants and young children are likely to be less dense in both nutrients and energy (most frequently consumed food was black tea, white bread and boiled rice) "These are the foods we grew up eating, we don't know other foods good for our young children". Some mothers felt that it is important for the child to gain good weight.

Feeding frequency was noted as a challenge. Most children were fed two to three times per day and the reasons being lack of fire woods for cooking. In addition, very low degree of dietary diversity in meals given to young children was observed (e.g. sardines are commonly eaten on almost daily basis) but children are given only stew. Children were rarely given vegetables because they would chock them. Responsive feeding was rarely practiced the reason being "If the child refuse to eat she/he must have eaten enough or she/he is not hungry". That means the child will definitely eat to satisfaction if she/he is hungry. In addition, most mothers reduced feeding amount and feeding frequency when a child falls sick saying the child refuses to eat and it is difficult to force a sick child to eat!

\section{Discussion}

In this study, decisions and practices regarding initiation of breastfeeding, exclusive and duration of breastfeeding were influenced by cultural values, family pressure, education, employment and place of delivery. Mothers/caregivers in this study were aware and majority practiced early initiation of breastfeeding, feeding colostrum and continued breastfeeding on demand. Although almost all surveyed mothers had breastfed their children, exclusive breastfeeding was still very low. Much lower exclusive breastfeeding practices observed in Unguja may be contributed by cultural beliefs that breast milk was not enough for the baby, mothers think the child feels thirst and sometimes water should be given to stop hiccups. In addition, mother's economic activities sometimes limited their availability to stay at home and breast feed their babies adequately and exclusively, hence early introduction of other foods. Since the mothers were also given porridge before they had attained the age of 6 months, they felt no harm doing the same to their infants. These are some of the contributing factors to early introduction of other foods to infant feeding regime. It is important for caregivers to understand that giving water or other foods tend to decrease breast milk production and perpetuates the notion that breast milk is not enough especially if this practice is done at an early age of the infant.

Mothers are faced with personal and social constraints in practicing exclusive breastfeeding. Specific constraints identified included maternal health, perceived milk insufficiency, inadequate breastfeeding skills, peer pressure and influence of other family members. The mismatch between breastfeeding intention and the practice of exclusive breastfeeding indicates the existence of knowledge gap for mothers, family members and health care providers. The study in Kigoma reported higher prevalence of exclusive breastfeeding (58\%) (Nkala \& Msuya 2011), which was higher than the National estimates (50\%) (TDHS, 2011). The observed differences could be a result of methods used to assess exclusive breastfeeding. Generally, the prevalence of exclusive breastfeeding globally is low (39\%) and it is estimated to be $36 \%$ in developing countries (UNICEF, 2007). Counselling and proper education on desirable breastfeeding practices should be adopted to achieve a change in attitudes, perceptions, knowledge and practice of exclusive breastfeeding (Agunbiade \& Ogunleye 2012).

Pre-lacteal feeds were given to infants and this is one of the sub-optimal breastfeeding practices, which was often practiced by mothers. Other sub-optimal practices included discarding of yellow milk (colostrum) within the first few days after delivery, infants given honey, herbal 
extracts, glucose solution, sugar, salt solution and infant formula within first few hours after delivery and the use of infant formula in first few days of infant's life. Various reasons were given for this practice. Apparently, these are practices that have been passed on from one generation to another; sometimes with limited understanding of the importance or consequences of the practices. Other surveys in Tanzania reported that one-third and a quarter of mothers give prelacteals in rural and urban areas, respectively (TDHS, 2011).

The timing of the introduction of complementary foods and drinks in addition to breast milk is very critical for child growth and development (Mamiro et al. 2005). Our findings showed that mean age for introduction of complementary foods was 4 months contrary to the WHO recommendations of 6 months (WHO, 2013). This is an indication that there are some mothers who start complementation at earlier age. Consequently, it reduces frequency of breastfeeding and amount of breast milk produced and consumed by the infant. This could contribute to the fear that most mothers have that they are not able to produce enough milk. It also exposes the infant to risk of contamination. Early initiation of complementary foods could probably be a contributing factor to higher rates of undernutrition in Zanzibar. Other studies done elsewhere showed that early initiation of complementary foods ranged from 0 to 4 months depending on several factors including geographical locality and ethnicity (Hussein, 2005; Nyaruhucha et al., 2006; Caulfield et al., 2006). Fluids given were water and thin cereal based porridge (Kulwa et al., 2006). A study by Victor and fellow researchers reported that risk factors for early initiation of complimentary feeding included low level of maternal education, limited access to mass media, lack of postnatal check-ups and low economic status (Nkala \& Msuya, 2011; Victor et al., 2014). Early introduction of complementary foods coupled with the serving of small meal sizes reflect inadequate nutrient intake.

Early exposure of children to food, nutrition and child care in schools would have an advantage of having future parents with adequate knowledge on caring practices. However, the current school curriculum in Zanzibar does not offer sufficient knowledge on these topics leaving majority of youth completing primary education with limited knowledge and skills on these aspects. It would be quite useful to introduce these subjects in the schools considering that many of these young fellows end up being parents immediately after completing their primary education with limited knowledge on feeding practices for themselves and for their off-springs.

Some limitations of the study include the fact that the survey was limited to the use of ProPAN tool and software in analysis which was adopted to our setting. The results could be different if another tool was used. Furthermore, the results of this study cannot be generalized to the whole of Zanzibar or to Tanzania mainland because of different cultural norms around child feeding practices.

In conclusion, infant and young child feeding in Zanzibar is suboptimal. Dietary inadequacies, inadequate care practices, use of pre-lacteals, early introduction of complementary foods and low rate of exclusive breastfeeding could probably be a contributing factor to higher rates of malnutrition. There is a need to strengthen IYCF counselling into the antenatal clinic regime and or establish community centres where women will be given information about child care and feeding. Effective communication through a combination of various communication channels including mobile phones, radio, schools and health workers is critical for behaviour change in improving IYCF practices.

\section{Acknowledgements}

The authors wish to thank UNICEF for financial support, Ministry of Health and Social Welfare, Zanzibar Medical Research Ethical Committee (ZAMREC) for permission and ethical clearance; the district leaders, Shehas of the surveyed Shehias and the mothers who participated in the study for their cooperation. We also appreciate the assistance of all health care workers. All authors read and approved the manuscript. No conflict of interest declared by any of the authors. 


\section{References}

Agunbiade, O.M. \& Ogunleye, O.V. (2012) Constraints to exclusive breastfeeding practice among breastfeeding mothers in Southwest Nigeria: implications for scaling up. International Breastfeeding Journal 7: 5-5.

Anderson, J.W., Johnstone, B.M. \& Remley, D.T. (1999) Breast-feeding and cognitive development: a meta-analysis. American Journal of Clinical Nutrition 70: 525-535.

Dewey, K.G., Cohen, R.J., Brown, K.H. \& Rivera, L.L. (2001) Effects of exclusive breastfeeding for four versus six months on maternal nutritional status and infant motor development: results of two randomized trials in Honduras. Journal of Nutrition 131: 262-267.

Hussein, A.K. (2005) Breastfeeding and complementary feeding in Tanzania. East African Journal of Public Health 2:27-32.

Kulwa, K.B.M., Mamiro, P.S., Kimanya, M.E., Mziray, R. \& Kolsteren, P.W. (2015) Feeding practices and nutrient content of complementary meals in rural central Tanzania: implications for dietary adequacy and nutritional status. BMC Pediatrics 15: 171.

Kulwa, K.B., Kinabo, J.L. \& Modest, B. (2006) Constraints on good child-care practices and nutritional status in urban Dar-es-Salaam, Tanzania. Food Nutrition Bulletin 27:236-244.

Caulfield, L.E., Richard, S.A., Rivera, J.A., Musgrove, P. \& Black, R.E. (2006) Stunting, Wasting, and Micronutrient Deficiency Disorders. In: Disease Control Priorities in Developing Countries 2nd edition. Washington (DC), World Bank.

Mamiro, P.S., Kolsteren, P., Roberfroid, D., Tatala, S., Opsomer, A.S. \& Van Camp, J.H. (2005). Feeding practices and factors contributing to wasting, stunting, and iron-deficiency anaemia among 3-23-month old children in Kilosa district, rural Tanzania. Journal of Health Population and Nutrition 23:222-230.

Motee, A. \& Jeewon, R. (2014) Importance of exclusive breastfeeding and complementary feeding among infants. Current Research on Nutrition and Food Sciences 2(2). doi : http://dx.doi.org/10.12944/CRNFSJ.2.2.02

TDHS (2011) Tanzania Demographic and Health Survey 2010. National Bureau of Statistics and ICF Macros, Dar es Salaam.

Nkala, T.E. \& Msuya, S.E. (2011) Prevalence and predictors of exclusive breastfeeding among women in Kigoma region, Western Tanzania: a community based cross-sectional study. International Breastfeeding Journal 6: 17.

Nyaruhucha, C.N., Msuya, J.M., Mamiro, P.S. \& Kerengi, A.J. (2006) Nutritional status and feeding practices of under-five children in Simanjiro District, Tanzania. Tanzania Health Research Bulletin 8:162-167.

PAHO (2004) Guiding Principles for Complementary Feeding of the Breastfed Child. Washington DC: Pan American Health Organization.

Stuebe, A. (2009) The risks of not breastfeeding for mothers and infants. Reviews in Obstetrics and Gynecology 2: 222-231.

UNICEF (2006) Progress for children: A report card on nutrition. Number 4. United Nation Children's Fund.

UNICEF (2007) Progress for Children: A World fit for Children. Statistical review, Number 6. United Nation Children's Fund. New York.

Victor, R., Baines, S.K., Agho, K.E. \& Dibley, M.J. (2014) Factors associated with inappropriate complementary feeding practices among children aged 6-23 months in Tanzania. Maternal and Child Nutrition 10:545-561.

WHO (2009) Infant and Young Child Feeding. World Health Organization.

WHO (2013) Essential nutrition actions: improving maternal, newborn, infant and young child health and nutrition. In World Health Organization (Press W ed. 20 Avenue Appia, 1211 Geneva 27, Switzerland.2013. 